\title{
A RESTRICTION THEOREM FOR SPACE CURVES
}

\author{
E. PRESTINI
}

Abstract. Let $\alpha$ be a $C^{k}$ curve $(k \geqslant 3)$ in $R^{3}$ with nonvanishing curvature and torsion. It is proved that the restriction operator $T:\left.f \rightarrow \hat{f}\right|_{\alpha}$ is bounded from $L^{p}\left(R^{3}\right)$ to $L^{q}(\alpha)$ if $1<p<15 / 13$ and $1 / q>6(1-1 / p)$, and that $T$ is not bounded if $p>6 / 5$ or $1 / q<6(1-1 / p)$.

Let $\alpha$ be a $C^{k}$ curve in $R^{3}(k \geqslant 3)$. For $f \in C_{0}^{\infty}\left(R^{3}\right)$ define the restriction operator $T$ by

$$
T f=\left.\hat{f}\right|_{\alpha}
$$

Then the following result holds:

THEOREM. If the curve $\alpha$ has nonvanishing curvature and torsion, the inequality

$$
\|T f\|_{L^{q}(\alpha)} \leqslant C_{p}\|f\|_{L^{p}\left(R^{3}\right)}
$$

holds if $1 \leqslant p<15 / 13$ and $1 / q>6(1-1 / p)$. The inequality does not hold if $p \geqslant 6 / 5$ or $1 / q<6(1-1 / p)$.

Proof. First we show that (1) does not hold if $1 / q<6(1-1 / p)$. Let $f$ be a smooth function such that its Fourier transform $\hat{f}$ is supported on a parallelepiped centered at any point $P$ on the curve and whose dimensions are $\eta, \eta^{2}, \eta^{3}$ along the tangent, normal and binormal direction at $P$, respectively ( $\eta$ denotes a small number). Then up to infinitesimals of higher order, $\|f\|_{L^{p}\left(R^{3}\right)}=\eta^{6(1-1 / p)}$ and $\|T f\|_{L^{q}(\alpha)}=\eta^{1 / q}$, hence $T$ cannot be a bounded operator unless $1 / q \geqslant 6(1-1 / p)$.

Now we prove (1). We may assume, without loss of generality, that $\alpha$ is defined by the equations $(t, \varphi(t), \psi(t), 0<t<\eta)$, where $\eta$ is a small number and $\varphi(t)=k t^{2} / 2+\xi(t), \psi(t)=k \tau t^{3} / 6+\zeta(t)$. Here $k$ and $\tau$ denote curvature and torsion, $\xi(t)$ and $\zeta(t)$ are infinitesimals of third and fourth order w.r.t. $t$. Then denote by $T^{*}$ the adjoint operator

$$
\begin{array}{r}
\left(T^{*} f\right)^{3}(y)=\int_{0}^{\eta} \int_{0}^{\eta} \int_{0}^{\eta} \exp \left(-i\left(y_{1}\left(\sum_{i} t_{i}\right)+y_{2}\left(\sum_{i} \varphi\left(t_{i}\right)\right)+y_{3}\left(\sum_{i} \psi\left(t_{i}\right)\right)\right)\right) \\
\cdot f\left(t_{1}\right) f\left(t_{2}\right) f\left(t_{3}\right) d t_{1} d t_{2} d t_{3}
\end{array}
$$

with $y=\left(y_{1}, y_{2}, y_{3}\right)$.

Received by the editors May 25, 1977.

AMS (MOS) subject classifications (1970). Primary 42A68, 42A72; Secondary 60E05.

○ American Mathematical Society 1978 
If we make the change of variables $u_{1}=\Sigma_{i} t_{i}, u_{2}=\Sigma_{i} \varphi\left(t_{i}\right), u_{3}=\Sigma_{i} \psi\left(t_{i}\right)$, we obtain

$$
\begin{array}{r}
\left(T^{*} f\right)^{3}(y)=\int_{D} \exp \left(-i\left(y_{1} u_{1}+y_{2} u_{2}+y_{3} u_{3}\right)\right) f\left(t_{1}\right) f\left(t_{2}\right) f\left(t_{3}\right) \\
\cdot\left|J\left(t_{1}, t_{2}, t_{3}\right)\right|^{-1} d u_{1} d u_{2} d u_{3},
\end{array}
$$

where $D$ denotes the image of the cube of side $\eta$ in the $\left(u_{1}, u_{2}, u_{3}\right)$-plane and $J$ the Jacobian of the transformation. Now we apply the Hausdorff-Young inequality and we change variables once more. In so doing we get

$$
\begin{aligned}
& \left\|T^{*} f\right\|_{L^{p^{*}}\left(R^{3}\right)}^{3}=\left\|\left(T^{*} f\right)^{3}\right\|_{L^{p^{*}\left(R^{3}\right)}} \\
& \leqslant C_{p}\left(\int_{D}\left(\left|f\left(t_{1}\right) f\left(t_{2}\right) f\left(t_{3}\right)\right|\left|J\left(t_{1}, t_{2}, t_{3}\right)\right|^{-1}\right)^{p^{*} /\left(p^{*}-3\right)} d u_{1} d u_{2} d u_{3}\right)^{\left(p^{*}-3\right) / p^{*}} \\
& =C_{p}\left(\int_{0}^{\eta} \int_{0}^{\eta} \int_{0}^{\eta}\left|f\left(t_{1}\right) f\left(t_{2}\right) f\left(t_{3}\right)\right|^{p^{*} /\left(p^{*}-3\right)}\right. \\
& \left.\cdot\left|J\left(t_{1}, t_{2}, t_{3}\right)\right|^{-p^{*} /\left(p^{*}-3\right)+1} d t_{1} d t_{2} d t_{3}\right)^{\left(p^{*}-3\right) / p^{*}}
\end{aligned}
$$

By the Vandermonde determinant

$$
\begin{aligned}
\left|J\left(t_{1}, t_{2}, t_{3}\right)\right| & =\frac{k^{2} \tau}{12}\left|\begin{array}{ccc}
1 & 1 & 1 \\
t_{1}+\xi^{\prime}\left(t_{1}\right) & t_{2}+\xi^{\prime}\left(t_{2}\right) & t_{3}+\xi^{\prime}\left(t_{3}\right) \\
t_{1}^{2}+\zeta^{\prime}\left(t_{1}\right) & t_{2}^{2}+\zeta^{\prime}\left(t_{2}\right) & t_{3}^{2}+\zeta^{\prime}\left(t_{3}\right)
\end{array}\right| \\
& >c\left|\left(t_{1}-t_{2}\right)\left(t_{1}-t_{3}\right)\left(t_{2}-t_{3}\right)\right| .
\end{aligned}
$$

Now set $a=3 /\left(p^{*}-3\right)$ and $F=|f|^{p^{*} /\left(p^{*}-3\right)}$. By the change of variables $v_{1}=t_{1}, v_{2}=t_{1}-t_{2}, v_{3}=t_{1}-t_{3}$, and by Hölder's inequality, we obtain

$$
\begin{array}{r}
\iiint F\left(v_{1}\right) F\left(v_{1}-v_{2}\right) F\left(v_{1}-v_{3}\right)\left|v_{2} v_{3}\left(v_{2}-v_{3}\right)\right|^{-a} d v_{1} d v_{2} d v_{3} \\
\quad \leqslant\|F\|_{L^{3}\left(R^{3}\right)}^{3} \iint\left|v_{2} v_{3}\left(v_{2}-v_{3}\right)\right|^{-a} d v_{2} d v_{3} \leqslant C_{1}\|F\|_{L^{3}\left(R^{3}\right)}^{3},
\end{array}
$$

when $a<2 / 3$. Hence

$$
\left\|T^{*} f\right\|_{L^{p^{*}}\left(R^{3}\right)}^{3} \leqslant C_{p}\|f\|_{L^{3 p^{*} /\left(p^{*}-3\right)}}^{3}(\alpha)
$$

for $p^{*}>15 / 2$. By duality this proves, in particular, the following inequality:

$$
\|T f\|_{L^{5 / 4+\varepsilon^{\prime}(\alpha)}} \leqslant C\|f\|_{L^{15 / 13-\xi^{*}\left(R^{3}\right)}}
$$

( $\xi^{\prime}$ and $\xi^{\prime \prime}$ denote small positive numbers). Now (1) follows by interpolating this estimate with the trivial one $\|T f\|_{L^{\infty}(\alpha)} \leqslant C_{2}\|f\|_{L^{1}\left(R^{3}\right)}$.

Finally, if in (2) we take $p^{*}=6$ and $f$ to be the constant 1 , using Plancherel's theorem we see that $T^{*} f$ does not belong to $L^{6}\left(R^{3}\right)$; hence $T$ is not a bounded operator from $L^{p}\left(R^{3}\right)$ to $L^{q}(\alpha)$ for any $q$ if $p \geqslant 6 / 5$. Note that the theorem does not take care of the case $1 / q>6(1-1 / p)$ and $15 / 13 \leqslant p<6 / 5$, or the case $1 / q=6(1-1 / p)$ and $1 \leqslant p \leqslant 15 / 13$. The 
reader should compare our techniques with those of [1] $[4]$.

I wish to thank my adviser C. Fefferman for suggesting the problem and guiding me during this research.

\section{BIBLIOGRAPHY}

1. C. Fefferman, Inequalities for strongly singular convolution operators, Acta Math. 124 (1970), 9-35.

2. __ A note on spherical summation multipliers, Israel J. Math. 15 (1973), 44-52.

3. L. Carleson and P. Sjolin, Oscillatory integrals and a multiplier problem for the disc, Studia Math. 44 (1972), 287-299.

4. P. Sjolin, Fourier multipliers and estimates of the Fourier transform of measures carried by smooth curves in $R^{2}$, Studia Math. 51 (1974), 169-182.

5. L. Hörmander, Oscillatory integrals and multipliers on FLP, Ark. Mat. 11 (1973), 1-11.

6. E. Stein and G. Weiss, Introduction to Fourier analysis on Euclidean spaces, Princeton Univ. Press, Princeton, N. J., 1971.

Departiment of Mathematics, Princeton University, Princeton, New Jersey 08540 